\title{
Claudin expression in rectal well-differentiated endocrine neoplasms (carcinoid tumors)
}

\author{
MITSUAKI ISHIDA $^{1}$, RYOJI KUSHIMA ${ }^{2}$ and HIDETOSHI OKABE ${ }^{1,2}$ \\ ${ }^{1}$ Department of Clinical Laboratory Medicine, ${ }^{2}$ Division of Diagnostic \\ Pathology, Shiga University of Medical Science, Shiga, Japan
}

Received June 24, 2008; Accepted September 24, 2008

DOI: 10.3892/or_00000197

\begin{abstract}
Claudins are the structures and functional components of tight junctions and have crucial roles in the maintenance of cell polarity, cellular arrangement, adhesion and paracellular transport. Various claudins are expressed in different epithelial cells and most tissues express multiple claudin proteins. The altered expression of claudins has been reported in various human carcinomas, but their expression in rectal well-differentiated endocrine neoplasms (carcinoid tumors), the most common endocrine tumors in the gastrointestinal tract, has yet to be examined. The expression of claudins-2, -3 and -4 in 16 rectal well-differentiated endocrine neoplasms was studied by immunohistochemical methods, and compared with their expression patterns in endocrine tumors of the pancreas and lung. According to previous reports, pancreatic endocrine tumors were positive for claudin- 3 and negative for claudins- 2 and -4 , and a majority of lung carcinoid tumors showed no immunoreactivity to claudins-2, -3 and -4 . However, our immunohistochemical study revealed that the rectal well-differentiated endocrine neoplasms showed diffuse positive immunoreactivity to claudins $-2,-3$ and -4 . These results indicate that claudin expression depends on the site of origin of endocrine tumors. In addition, claudin-3 and -4 expression in rectal welldifferentiated endocrine neoplasms suggests the possibility of a new therapeutic strategy. Claudins- 3 and -4 are receptors for the cytotoxic Clostridium perfringens enterotoxin. This enterotoxin rapidly and specifically lyses cells expressing claudins-3 and -4 and has a potential application in cancer therapeutics. Accordingly, this enterotoxin may be applicable for the treatment of rectal well-differentiated endocrine neoplasms in the future in order to prevent unexpected metastatic recurrences after tumor resections, because these
\end{abstract}

Correspondence to: Dr Hidetoshi Okabe, Department of Clinical Laboratory Medicine and Division of Diagnostic Pathology, Shiga University of Medical Science, Tsukinowa-cho, Seta, Otsu, Shiga 520-2192, Japan

E-mail: okabe@belle.shiga-med.ac.jp

Key words: well-differentiated endocrine neoplasm, carcinoid tumor, claudin, rectum neoplasms have a relatively high incidence of metastases despite their small size.

\section{Introduction}

Claudins have recently been identified as a 24-gene family of structures and functional components of the tight junctions (TJs) in the epithelial and endothelial cells $(1,2)$. TJs have crucial roles in maintaining cell polarity, cellular arrangement, adhesion and paracellular transport $(1,2)$. Various claudins are expressed in different epithelial cells and most tissues express multiple claudin proteins $(1,2)$. This is thought to account for the variability of different tissue functions.

Claudins have become a prominent subject in cancer research. Alterations in the claudin expression are believed to play an important role in tumorigenesis and tumor progression. An altered expression of claudins has been shown for various human neoplasms, including colorectal, breast, ovarian, pancreatic, lung and prostate carcinomas (3-9).

For example, claudins- 3 and -4 are up-regulated in the large population of colorectal (3), breast carcinomas (4) and ovarian adenocarcinomas (5). In the ductal adenocarcinomas of the pancreas, claudins- 2 and -4 are up-regulated, but claudin-3 is not expressed (6). A strong expression of claudins- 3 and -4 has been detected in prostatic adenocarcinomas (7), and the claudin-3 expression was correlated with advanced stage and recurrence, while that of claudin- 4 was correlated with advanced stage (8).

Although the claudin expression has been reported in the endocrine tumors of pancreas (6) and lung (9), it has yet to be studied in rectal well-differentiated endocrine neoplasms (carcinoid tumors), which constitute the most common endocrine neoplasms in the gastrointestinal tract. This study aimed to clarify the expression and localization of claudins in normal colorectal mucosae and in rectal well-differentiated endocrine neoplasms. The difference and/or similarity of claudin expression between rectal well-differentiated endocrine neoplasms and endocrine tumors of the pancreas and lung are discussed. Additionally, information obtained on claudin localization offers a significant potential for therapeutic use.

\section{Materials and methods}

Tissue specimens of rectal well-differentiated endocrine neoplasms (carcinoid tumors). Sixteen formalin-fixed, 
Table I. Clinical features and claudin expression of the rectal well-differentiated endocrine neoplasms (carcinoid tumors).

\begin{tabular}{ccccc}
\hline Case no. & Age/gender & Claudin-2 & Claudin-3 & Claudin-4 \\
\hline 1 & $66 / \mathrm{M}$ & $5, \mathrm{c}$ & $5, \mathrm{~m}$ & $5, \mathrm{~m}$ \\
2 & $54 / \mathrm{M}$ & $5, \mathrm{c}$ & $5, \mathrm{~m}$ & $4, \mathrm{~m}$ \\
3 & $36 / \mathrm{M}$ & $5, \mathrm{c}$ & $5, \mathrm{~m}$ & $5, \mathrm{~m}$ \\
4 & $57 / \mathrm{M}$ & $5, \mathrm{c}$ & $5, \mathrm{~m}$ & $5, \mathrm{~m}$ \\
5 & $81 / \mathrm{M}$ & $5, \mathrm{c}$ & $5, \mathrm{~m}$ & $5, \mathrm{~m}$ \\
6 & $34 / \mathrm{M}$ & $5, \mathrm{c}$ & $5, \mathrm{~m}$ & $4, \mathrm{~m}$ \\
7 & $51 / \mathrm{F}$ & $5, \mathrm{c}$ & $5, \mathrm{~m}$ & $5, \mathrm{~m}$ \\
8 & $41 / \mathrm{F}$ & $5, \mathrm{c}$ & $5, \mathrm{~m}$ & $3, \mathrm{~m}$ \\
9 & $29 / \mathrm{M}$ & $5, \mathrm{c}$ & $5, \mathrm{~m}$ & $4, \mathrm{~m}$ \\
10 & $41 / \mathrm{M}$ & $5, \mathrm{c}$ & $5, \mathrm{~m}$ & $3, \mathrm{~m}$ \\
11 & $43 / \mathrm{M}$ & $5, \mathrm{c}$ & $4, \mathrm{~m}$ & $3, \mathrm{~m}$ \\
12 & $26 / \mathrm{M}$ & $5, \mathrm{c}$ & $5, \mathrm{~m}$ & $4, \mathrm{~m}$ \\
13 & $69 / \mathrm{M}$ & $5, \mathrm{c}$ & $5, \mathrm{~m}$ & $5, \mathrm{~m}$ \\
14 & $57 / \mathrm{M}$ & $5, \mathrm{c}$ & $5, \mathrm{~m}$ & $5, \mathrm{~m}$ \\
15 & $53 / \mathrm{F}$ & $5, \mathrm{c}$ & $5, \mathrm{~m}$ & $5, \mathrm{~m}$ \\
16 & $65 / \mathrm{F}$ & $5, \mathrm{c}$ & $5, \mathrm{~m}$ & $5, \mathrm{~m}$ \\
\hline
\end{tabular}

0, $0-5 \%$ positive; $1,6-20 \%$ positive; $2,21-40 \%$ positive; $3,41-60 \%$ positive; $4,61-80 \%$ positive; $5,81-100 \%$ positive; $\mathrm{m}$, membranous staining and c, cytoplasmic staining.

paraffin-embedded tissue specimens from consecutive rectal well-differentiated endocrine neoplasms (carcinoid tumors) were obtained from 16 patients in our hospital during 20042007. The median age of the patients (12 males and 4 females) was 50.2 years (range 26-81) (Table I). The specimens were re-evaluated by at least two pathologists according to the criteria described in the World Health Organization Classification of Tumors, Pathology and Genetics of Tumors of the Digestive System (10) and Histological Typing of Endocrine Tumors (11).

Elastica van Gieson staining was performed in addition to hematoxylin and eosin staining for the evaluation of angioinvasion. Ten non-neoplastic colorectal mucosae attached to the resected tumors were also analyzed.

Immunohistochemistry. Deparaffinized 3- $\mu \mathrm{m}$ sections of the specimens were processed for the LSAB system (Dako Japan Co. Ltd., Kyoto, Japan) using the primary antibodies: claudin-2 monoclonal mouse (diluted 1:100; 12H12, Zymed Laboratories Inc., San Francisco, CA, USA), claudin-3 polyclonal rabbit (diluted 1:100; Zymed), claudin-4 monoclonal mouse (diluted 1:100; 3E2C1, Zymed), synaptophysin monoclonal mouse (diluted $1: 200 ; 27$ G12, Novocastra Laboratories Ltd., Newcastle upon Tyne, UK), chromogranin A monoclonal mouse (diluted 1:50; LK2H10, Novocastra) and Ki-67 monoclonal mouse antibody (diluted 1:50; MM1, Novocastra). Antigen retrieval was conducted by $20 \mathrm{~min}$ of incubation in $0.01 \mathrm{M}$ citrate buffer $(\mathrm{pH}$ 6.0) in a microwave oven at $500 \mathrm{~W}$. The sections were then washed with $0.1 \mathrm{M}$ phosphate-buffered saline ( $\mathrm{pH}$ 7.4) and bathed in $3 \% \mathrm{H}_{2} \mathrm{O}_{2}$ in methanol for $20 \mathrm{~min}$ at room temperature to quench endogenous peroxidase activity. Subsequently, the primary antibodies were applied to the sections, and incubated overnight in a humidified chamber at $4^{\circ} \mathrm{C}$. After three washing steps in phosphate-buffered saline, sections were incubated with biotinylated secondary antibody for $20 \mathrm{~min}$ at room temperature and sequentially stained using a $0.1 \%$ peroxidase-conjugated streptavidin solution, according to the manufacturer's instructions. Sections were then incubated in 3,3'-diaminobenzidine tetrahydrochloride (DAB; Nichirei) for a few minutes and counterstained with hematoxylin. The localization of immunohistochemical stainings for claudins and other markers was carried out in the serial sections to determine whether any correlations could be established between the marker distributions in the tumor in this study.

Evaluation of immunoreactivity. Immunohistochemical findings were verified by control studies. Negative controls consisted of slides run without the primary antibodies. Specificity of the immunoreactivity to individual antibodies was evaluated by inner and outer positive controls. Nonneoplastic colorectal mucosae within the tumor tissue slides were used as the inner positive controls for the immunoreactivity to claudins -3 and -4 . Pancreatic ductal adenocarcinoma was used as the outer positive control for claudins-2 and -4 , and pancreatic endocrine tumor was used as the outer positive control for claudin-3 (6). Non-neoplastic colorectal mucosal endocrine cells within the tumor tissue slides were used as the inner positive controls for synaptophysin and chromogranin A. Lymph node sections were used as the external positive control for Ki-67.

Immunoreactivity to claudins was scored semi-quantitatively according to the method of Borka et al (6) and Moldvay et al (9). Ten randomly selected areas of each tissue slide were analyzed using high-power field (x400) in counting 100 cells per field. Immunoreactivity was evaluated by the percentage of cells staining positively. The values scored for semi-quantitative evaluation were: 0 (0-5\% positive), 1 (6-20\% positive), 2 (21-40\% positive), 3 (41-60\% positive), 4 (61-80\% positive) and 5 (81-100\% positive). Positive reactions were scored by membranous or cytoplasmic staining.

Immunoreactivity to synaptophysin and chromogranin A was scored as positive or negative. Ki-67 labeling indices were evaluated by counting the percentage of positive cells in 1,000 tumor cells.

\section{Results}

Clinicopathological features of well-differentiated endocrine neoplasms (carcinoid tumors). A well-differentiated endocrine neoplasm (carcinoid tumor) is defined as a neoplasm of monomorphous endocrine cells showing mild or no atypia and growing as solid nests, trabeculae or pseudoglands (Fig. 1). Our 16 cases were restricted to the mucosa or submucosa without angioinvasion, as evaluated by elastica van Gieson staining, and without metastases to the lymph nodes and other visceral organs, including the liver.

Claudin expression in normal colorectal mucosae. Claudin-2 expression was barely detectable or absent in the normal 


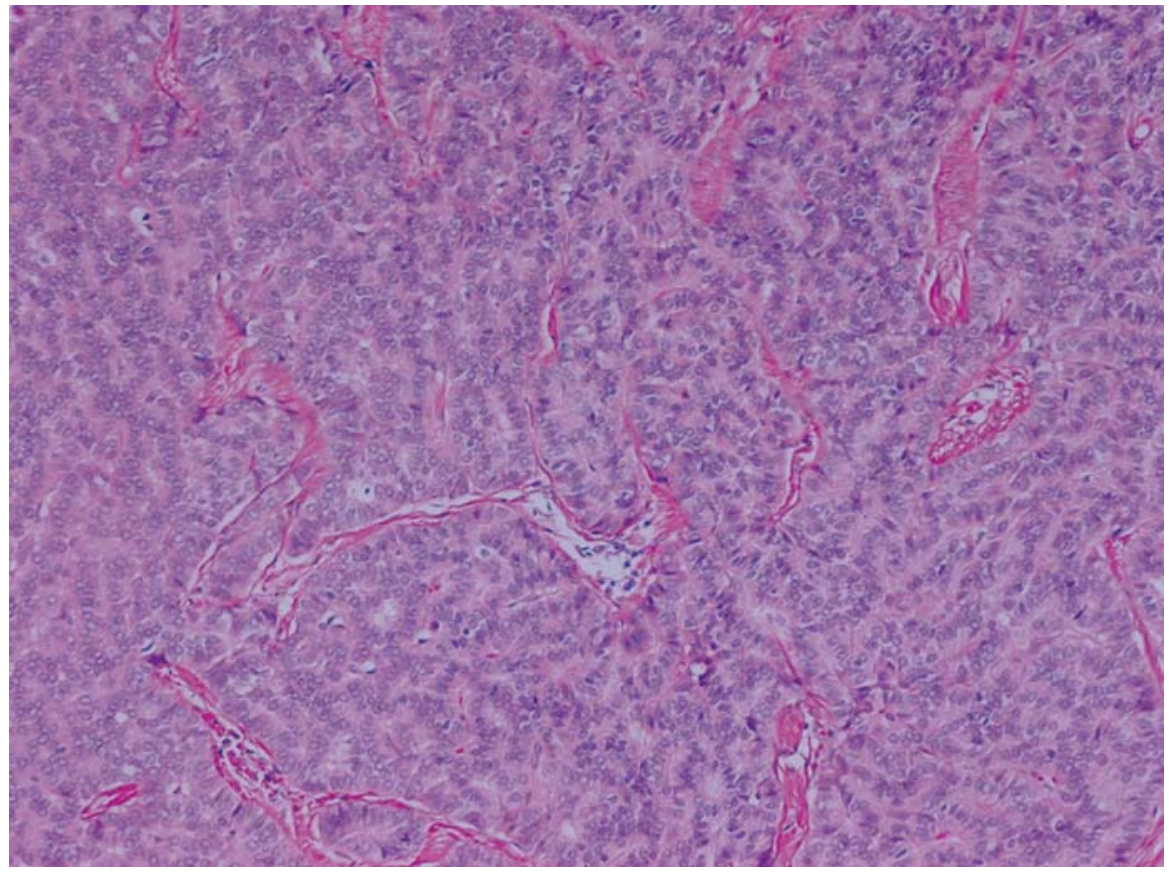

Figure 1. Well-differentiated endocrine neoplasm (carcinoid tumor) of the rectum (Case 15), H\&E (original magnification, x100). A typical well-differentiated endocrine neoplasm showing the trabecular, insular growth of uniform neuroendocrine cells.
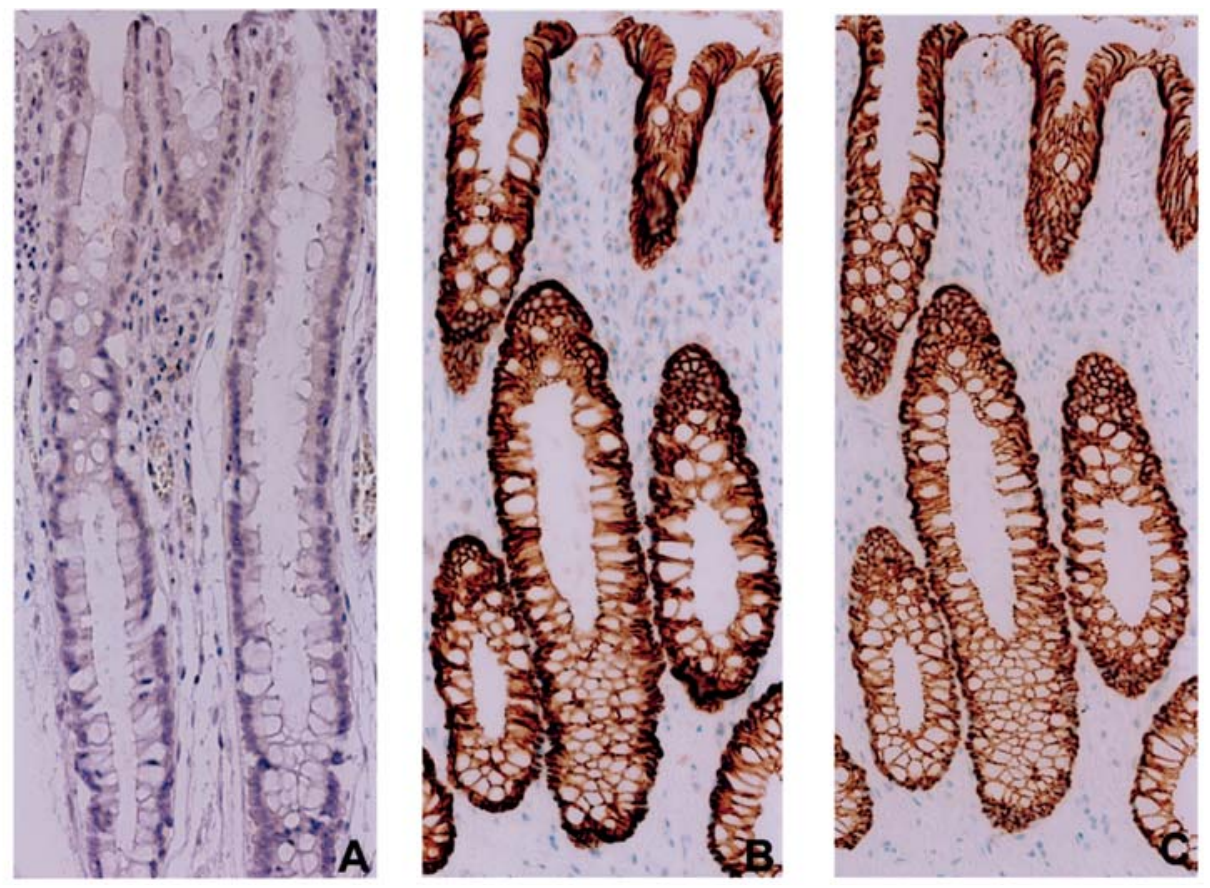

Figure 2. Immunohistochemical staining of normal colorectal mucosae. (A) Claudin-2 immunoreactivity is absent. (B) Claudins-3 (C) and -4 are strongly expressed in the colorectal epithelial membranes (original magnification, $\mathrm{x} 100$ ).

colorectal mucosae (Fig. 2A). Claudins-3 and -4 were detected in the epithelial membranes (Fig. 2B and C).

Synaptophysin and chromogranin A expression and Ki-67 labeling indices in rectal well-differentiated endocrine neoplasms. Synaptophysin was expressed in the rectal welldifferentiated endocrine neoplasms (16/16 cases). Chromogranin A was positive in 5 cases (5/16). Ki-67 labeling indices were $<1 \%$ in the 16 cases.
Claudin expression in rectal well-differentiated endocrine neoplasms. Table I summarizes claudin expression in 16 rectal well-differentiated endocrine neoplasms. Claudin-2 was diffusely expressed in the cytoplasm of tumor cells in the 16 cases (mean score 5.00) (Fig. 3A) and no membranous staining was found. Claudins- 3 and -4 displayed membranous immunoreactivity in the 16 cases (mean score 4.94 and 4.38 , respectively) (Fig. 3B and C) and no cytoplasmic staining was found. 

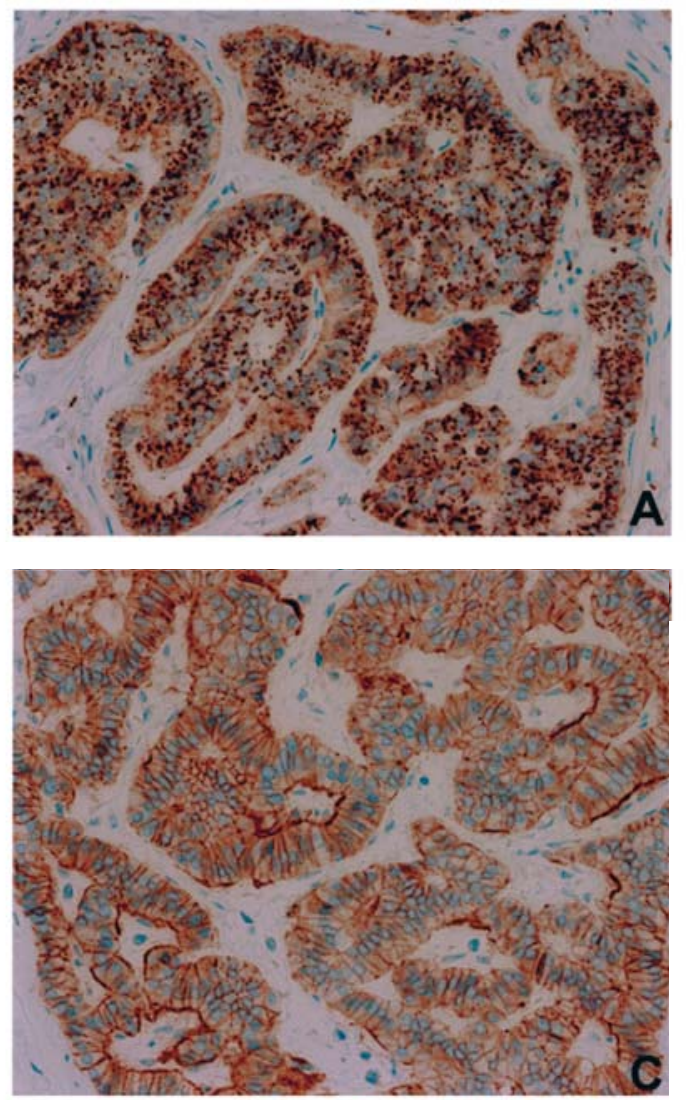

Figure 3. Immunohistochemical results of well-differentiated endocrine neoplasm (carcinoid tumor) (Case 15). (A) Claudin-2 is diffusely expressed in the cytoplasm of the tumor cells. (B) Claudins-3 and (C) -4 are strongly expressed in the cell membranes of the tumor cells (original magnification, x200).

\section{Discussion}

Claudin expression varies considerably in individual endocrine tissues and neoplasms $(6,9)$. Borka et al reported that normal pancreatic endocrine cells and tumors were positive for claudin-3, but negative for claudins-2 and -4 (6). Moldvay et al revealed that the vast majority of carcinoid tumors of the lung lacked immunoreactivity to claudins-2, -3 and -4 (mean immunoreactivity scores according to the same methods as our study: $1.15,0.62$ and 0.69 , respectively). However, small cell carcinomas showed positive immunoreactivity to claudins-3 and -4 (mean 4.40 and 3.87 , respectively), but claudin-2 was not expressed (mean 0) (9). Our immunohistochemical study showed that the rectal well-differentiated endocrine neoplasms showed positive immunoreactivity to claudins-2, -3 and -4 (mean 5.00, 4.94 and 4.38, respectively). These results reveal that claudin expression depends on the site of origin of endocrine tumors. In addition, synaptophysin was expressed in our 16 well-differentiated endocrine neoplasms, but chromogranin A was expressed only in 5 cases $(5 / 16)$. These results are consistent with our previous studies $(12,13)$ as regards the low incidence of chromogranin A expression in rectal well-differentiated endocrine neoplasms.

Claudin-2 is a TJ component in many tissues including the liver and kidney. It is considered to form ion-selective channels in the TJs of epithelial cells and to have an important

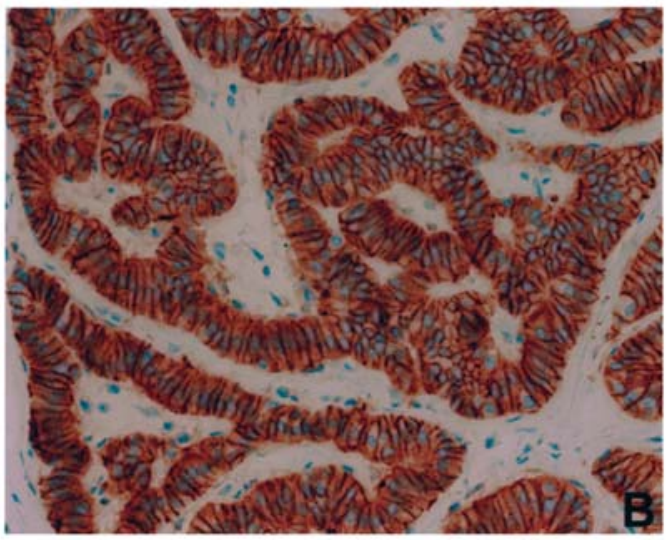

role in the paracellular barrier function (14). Claudin-2 is not expressed in the normal colorectal and gastric mucosae including intestinal metaplastic mucosae $(15,16)$. However, a strong claudin-2 expression is observed in colorectal epithelia affected by active ulcerative colitis and Crohn's disease (15). Song et al reported that the large population of the gastric intestinal type adenocarcinomas was strongly positive for claudin-2. They also stated that claudin-2 expression is closely correlated with gastric carcinogenesis (16). Our immunohistochemical study revealed that claudin-2 was diffusely expressed in the cytoplasm of the rectal well-differentiated endocrine neoplasms and that the aberrant claudin- 2 expression in rectal well-differentiated endocrine neoplasms may be related to their tumorigenesis as suggested for gastric intestinal type adenocarcinoma (16).

Claudins- 3 and -4 are TJ components, expressed in many normal organs and overexpressed in several carcinomas, including those of the breast, ovary, prostate and pancreas (3-5,7). Claudins-3 and -4 are receptors for the cytotoxic Clostridium perfringens enterotoxin (CPE) $(17,18)$. CPE is known to injure intestinal epithelial cells by increasing membrane permeability leading to loss of osmotic equilibrium and subsequent cytolysis and cell death (19). Interestingly, CPE has emerged as a molecular target in the therapy of malignant tumors, as it rapidly and specifically lyses cells expressing claudins-3 and -4 . Thus, these molecules can be exploited for the treatment of tumors in which their consistent expression is known. Rectal well-differentiated endocrine neoplasms, which are the most common endocrine tumors in the gastrointestinal tract, have a relatively high incidence of lymph node and liver metastases despite their small-sized lesions (20). To prevent unexpected metastases after local tumor resections, CPE therapy may be appropriate because our immunohistochemical study revealed claudins-3 and -4 were strongly expressed in the 16 rectal well-differentiated endocrine neoplasms.

Our provisional study revealed that claudins-3 and/or -4 were expressed in $4 / 5$ cases of colorectal small cell carcinomas (poorly differentiated neuroendocrine carcinomas) (unpublished data). Small cell carcinoma is highly aggressive and has a high incidence of multiple metastases. Moreover, the strategy of CPE therapy appears to be applicable for its treatment in the future. However, since small cell carcinoma did not always have claudins- 3 and/or -4 (4/5 cases) as shown in our preliminary study, further immunohistochemical 
analyses of claudins- 3 and -4 will be needed before initiating a trial of CPE therapy.

Systemic toxicity presents an important problem for CPE therapy, because other sites, such as the normal gastrointestinal tract, lung and prostate also express claudins- 3 and -4 . Thus systemic delivery of CPE would result in significant toxicity such as immobility and loss of appetite (21). However, a local delivery method was reported to be effective in treating xenografts of cancer cells in mice by Kominsky et al (4). Further studies are needed for the practical application of CPE therapy for the treatment of carcinomas, including the neuroendocrine tumors.

\section{Acknowledgements}

This research was supported in part by Grants-in-Aid for Young Scientists (B) (no. 20790281).

\section{References}

1. Van Itallie CM and Anderson JM: Claudins and epithelial paracellular transport. Annu Rev Physiol 68: 403-429, 2006.

2. Krause G, Winkler L, Mueller SL, Haseloff RF, Piontek J and Blasig IE: Structure and function of claudins. Biochim Biophys Acta 1778: 631-645, 2007.

3. de Oliveira SS, de Oliveira IM, De Souza W and Morgado-Diaz JA: Claudins upregulation in human colorectal cancer. FEBS Lett 579: 6179-6185, 2005.

4. Kominsky SL, Vali M, Korz D, et al: Clostridium perfringens enterotoxin elicits rapid and specific cytolysis of breast carcinoma cells mediated through tight junction proteins claudin 3 and 4. Am J Pathol 164: 1627-1633, 2004.

5. Zhu Y, Brännström M, Janson PO and Sundfeldt K: Differences in expression patterns of the tight junction proteins, claudin 1,3 , 4 and 5 , in human ovarian surface epithelium as compared to epithelia in inclusion cysts and epithelial ovarian tumors. Int J Cancer 118: 1884-1891, 2006.

6. Borka K, Kaliszky P, Szabo E, et al: Claudin expression in pancreatic endocrine tumors as compared with ductal adenocarcinomas. Virchows Arch 450: 549-557, 2007.

7. Väre P, Loikkanen I, Hirvikoski P, Vaarala MH and Soini Y: Low claudin expression is associated with high Gleason grade in prostate adenocarcinoma. Oncol Rep 19: 25-31, 2008.

8. Sheehan GM, Kallakury BVS, Sheehan CE, Fisher HAG, Kaufman RP and Ross JS: Loss of claudins- 1 and -7 and expression of claudins-3 and -4 correlate with prognostic variables in prostatic adenocarcinomas. Hum Pathol 38: 564-569, 2007.
9. Moldvay J, Jäckel M, Paska C, Soltesz I, Schaff Z and Kiss A: Distinct claudin expression profile in histologic subtypes of lung cancer. Lung Cancer 57: 159-167, 2007.

10. Capella C, Solica E, Sobin LH and Arnold R: Endocrine tumors of the colon and rectum. In: World Health Organization Classification of Tumors, Pathology and Genetics of Tumors of the Digestive System. Hamilton SR and Aaltonen LA (eds). IARC Press, Lyon, pp137-139, 2000.

11. Solica E, Klöppel G and Sobin LH: Endocrine tumors of the gastrointestinal tract. In: Histology Typing of Endocrine Tumors.. Solica E, Klöppel G and Sobin LH (eds). 2nd edition Springer-Verlag, Berlin, pp61-69, 2000.

12. Ishida M, Kushima R, Chano T and Okabe H: Immunohistochemical demonstration of the type III intermediate filament peripherin in human rectal mucosae and well-differentiated endocrine neoplasms. Oncol Rep 18: 633-637, 2007.

13. Ishida M, Kushima R, Brevet M, Chatelain D and Okabe H: Coexpression of neuronal intermediate filaments, peripherin and alpha-internexin in human well-differentiated endocrine neoplasms (carcinoid tumors) of the appendix. Mol Med Rep 1: 191-195, 2008

14. Sakaguchi T, Gu X, Golden HM, Suh ER, Rhoads DB and Reinecker HC: Cloning of the human claudin-2 5'- flanking region revealed a TATA-less promoter with conserved binding sites in mouse and human for caudal-related homeodomain proteins and hepatocyte nuclear factor-1 alpha. J Bio Chem 277: 21361-21370, 2002.

15. Prasad S, Mingrino R, Kaukinen K, et al: Inflammatory processes have differential effects on claudins 2, 3 and 4 in colonic epithelial cells. Lab Invest 85: 1139-1162, 2005.

16. Song X, Li X, Tang Y, et al: Expression of claudin-2 in the multistage process of gastric carcinogenesis. Histol Histopathol 23: 673-682, 2008

17. Fujita K, Katahira J, Horiguchi Y, Sonoda N, Furuse M and Tsukita S: Clostridium perfringens enterotoxin binds to the second extracellular loop of claudin-3, a tight junction integral membrane protein. FEBS Lett 476: 258-261, 2000.

18. Katahira J, Inoue N, Horiguchi Y, Matsuda M and Sugimoto N: Molecular cloning and functional characterization of the receptor for Clostridium perfringens enterotoxin. J Cell Biol 136: 1239-1247, 1997

19. McClane BA and McDonel JL: Protective effects of osmotic stabilizers on morphological and permeability alterations induced in Vero cells by Clostridium perfringens enterotoxin. Biochim Biophys Acta 641: 401-409, 1981.

20. Soga J: Early-stage carcinoids of the gastrointestinal tract: an analysis of 1914 reported cases. Cancer 103: 1587-1595, 2005.

21. Wallace FM, Mach AS, Keller AM and Lindsay JA: Evidence for Clostridium perfringens enterotoxin (CPE) inducing a mitogenic and cytokine response in vitro and a cytokine response in vivo. Curr Microbiol 38: 96-100, 1999. 